\section{Calidad del servicio en residencias para mayores. Análisis de un caso en la Comunidad Valenciana}

\section{Quality of Service in Residences for the Elderly. Analysis of a Case in the Valencian Community}

\author{
Aldo Giovanni Caypa Altare ${ }^{1, *}$ \\ Ana Redondo Cano ${ }^{2}$ \\ 1. Máster Gestión de la Calidad. Especialidad Senvicios y Producción. Facultad de Economía. \\ Universidad de Valencia. Valencia. España. \\ 2. PDI Titular. Universidad de Valencia. Coordinadora Titulación de Grado. Valencia. España.
}

*Autor para correspondencia.

Correo electrónico: alcayal@alumni.uv.es (Aldo Giovanni Caypa Altare).

Recibido el 7 de junio de 2018; aceptado el 13 de mayo de 2019.

\section{RESUMEN}

Las residencias para adultos mayores se han convertido en un servicio clave y cada día más demandado por las personas de la tercera edad que por diferentes razones terminan viviendo en estas instituciones. Por ese motivo, conocer cuál es el grado de satisfacción de sus residentes se convierte en un aspecto clave para la mejora de la calidad en el servicio. Este estudio evaluó

la calidad del servicio prestado a los residentes de una residencia privada para mayores mediante la aplicación de una encuesta de satisfacción, basada en el modelo SERVPERF, donde se evaluó cada una de sus cinco dimensiones: elementos tangibles, fiabilidad, capacidad de respuesta, seguridad y empatía, y se agregó una nueva, la alimentación. Para la evaluación de los resultados se llevó a cabo análisis descriptivo, distribución de frecuencias, medias y desviación estándar, en una muestra de 58 adultos, durante los meses de mayo y junio de 2017. En general, se evidenció que los residentes se encontraban satisfechos con el servicio ofrecido; entre los aspectos positivos destacaban las habitaciones, los empleados y los horarios de visitas. Entre las causas de insatisfacción más importantes figuraron la alimentación y el miedo a sentir que puedan robarle sus enseres.

PALABRAS CLAVE: SERVPERF, calidad, adultos, residencias.

\section{ABSTRACT}

Residences for the elderly have become a key service and are increasingly in demand by the elderly, who for different reasons end up living there; for this reason, knowing the degree of satisfaction of its residents becomes a key aspect in improving the quality of the service. This study evaluated the quality of the service provided to residents of a private residence for the elderly, through the application of a satisfaction survey, based on the SERVPERF model, which consisted of five dimensions: tangibles, reliability, responsiveness, assurance and empathy, as well as a new one, feeding. In order to evaluate the results, frequency distribution, means and standard deviation were carried out using a sample of 58 adults between may and june 2017. It could be seen, that the residents were generally satisfied with the service offered, in particular, the rooms, the employees and the visiting hours, although among some major causes of dissatisfaction were food and the fear of having their belongings stolen.

KEYWORDS: SERVPERF, quality, elderly, residences.

\section{- INTRODUCCIÓN}

A lo largo del tiempo, la calidad en la prestación de los servicios se ha tornado en un proceso clave, en el cual el cliente tiene un papel vital ${ }^{1}$. Medir la calidad del servicio se convierte en una herramienta básica que las empresas deben implantar para conocer cuál es el grado de satisfacción que tienen sus usuarios con respecto a los servicios ofertados ${ }^{2}$.

Se han desarrollado diferentes modelos para medir la satisfacción de los usuarios. Entre ellos se encuentra el modelo SERVQUAL ${ }^{3}$. Este modelo, que mide las expectativas y las percepciones de los usuarios respecto a los servicios ofertados, ha sido ampliamente utilizado en diferentes tipos de servicios.

Algunos autores plantean ciertas limitaciones del SERVQUAL. Sostienen que la percepción por sí sola proporciona un mejor poder predictivo que si se consideran expectativas y percepciones. Así mismo, las puntuaciones obtenidas de la evaluación de la percepción muestran mejor fiabilidad y validez que aquellas obtenidas de la diferencia entre expectativas y percepciones $s^{4,5}$, por lo que se decantan por el uso de otro modelo como es el SERVPERF'. Mediante este modelo se mide únicamente la percepción de los usuarios, y también ha sido utilizado extensamente en diferentes tipos de servicios.

En los últimos años, en España se ha visto un incremento en la población de la tercera edad según datos del Patrón Continuo (INE, Instituto Nacional de Estadística) a 1 enero de 2016; el 18,4\% sobre el total de la población eran adultos mayores; a su vez, en 2066, según proyección del INE, un 34,6\% del total de la población corresponderá a personas de la tercera edad ${ }^{7}$, lo que sugiere la importancia de establecer servicios encaminados a suplir sus necesidades y expectativas.

Las residencias para mayores se han transformado en una opción para aquellas personas que, por diferentes razones, como una edad avanzada, soledad o estado de salud, entre otras, deciden irse a vivir de forma voluntaria a este tipo de residencia, dadas las características que ofrece ${ }^{8}$. La importancia actual y proyección futura de estos servicios, conocer cuál es el grado de satisfacción de sus residentes con respecto a la atención ofrecida, es un punto crucial para la mejora del servicio ofrecido. Por tal motivo, las encuestas de satisfacción 
se han convertido en una herramienta indispensable para medir la satisfacción de sus usuarios ${ }^{9-11}$.

La falta, en general, del desarrollo de instrumentos para medir la satisfacción en las residencias para mayores, así como el consenso de quiénes son sus clientes, con qué frecuencia se realizan las mediciones y qué criterios deberían ser tenidos en cuenta para seleccionar a los residentes para los estudios de satisfacción ${ }^{12}$ hacen imprescindible establecer un instrumento mediante el cual se pueda evaluar el grado de satisfacción de las personas de la tercera edad que se encuentren viviendo en las residencias para mayores, en relación con el servicio ofrecido. Por este motivo, este estudio pretende medir el grado de satisfacción de las personas de la tercera edad en residencias para mayores, con la aplicación del modelo SERVPERF' ${ }^{6}$, justificado por sus ventajas de aplicación: el cuestionario empleado, la sencillez y facilidad de interpretación y análisis de la información ${ }^{13,14}$. El modelo SERVPERF requiere de la construcción de una encuesta de satisfacción, a través de la cual se pueda obtener información integral y precisa sobre la percepción del servicio recibido, destacándose aquellos aspectos que causan satisfacción e insatisfacción en sus residentes, para que a partir de ellos se establezcan mejoras que propicien el cambio de directrices, comportamientos, hábitos, situaciones y aspectos en general, que incrementen la satisfacción de los residentes.

\section{- OBJETIVO GENERAL}

Evaluar la calidad del servicio prestado a los residentes de las residencias para mayores, basados en el modelo SERVPERF.

\section{- OBJETIVOS ESPECÍFICOS}

- Determinar qué causas generan mayor satisfacción en residentes de las residencias para mayores, con respecto a la atención ofrecida.

- Establecer cuáles son las principales causas que generan insatisfacción en las residencias para mayores durante la prestación del servicio a sus residentes.

- Identificar posibles áreas de mejora en relación con la atención recibida en residencias para mayores.

\section{- METODOLOGÍA}

De forma aleatoria, se escogió una residencia que alberga a personas mayores dependientes, donde prevaleció para su selección que fuera de carácter privada, ubicada en un municipio de la Comunidad Valenciana, y que contara con el apoyo y disposición de sus directivos, personal administrativo, sanitario y residentes para el desarrollo del estudio.
Se contó con una muestra de 58 mayores; para su obtención se tuvieron en cuenta los siguientes criterios de inclusión: mayores que se encontraban viviendo en la residencia, mayores de ambos sexos y mayores que fueron clasificados como A y B, según el examen cognitivo Mini Examen Cognitivo de Lobo (MEC), herramienta válida y fiable, para la evaluación del desarrollo cognitivo en la población de la tercera edad ${ }^{15}$.

Se diseñó un instrumento, la "encuesta de satisfacción", para la obtención de la información, con preguntas cerradas. El cuestionario se dividió en cuatro partes: la primera constó de una pregunta a desarrollar en el instrumento, para establecer el motivo por el cual las personas de la tercera edad se encuentran en las residencias. En la segunda parte se ponderaron los 6 atributos o dimensiones, de acuerdo con el grado de importancia dado por el encuestado, tenidos en cuenta en el estudio, como son:

1. La apariencia física, comodidad y mantenimiento de las instalaciones y materiales físicos.

2. Capacidad que tiene la residencia de cumplir de forma segura y precisa lo prometido.

3. Capacidad de respuesta que tienen los empleados para brindarle un servicio rápido.

4. Conocimiento y cortesía de los empleados y su habilidad para inspirar confianza y seguridad.

5. Empatía, cuidado y atención individualizada ofrecida por los empleados de la residencia.

6. Alimentación, variedad de los alimentos y bebidas, así como la disponibilidad de menús adaptados a las necesidades de los residentes.

Se organizaron por orden de importancia de 1 a 6 , siendo uno (1) la de menor importancia y seis (6) la de mayor importancia.

En la tercera parte se fijan los parámetros, establecidos en el modelo SERVPERF $F^{6}$, donde se incluyeron los ítems que contiene la encuesta con la realización de preguntas cerradas, por cada una de sus dimensiones, como son: elementos tangibles (3 preguntas), fiabilidad (4 preguntas), capacidad de respuesta (4 preguntas), seguridad (5 preguntas) y empatía ( 4 preguntas). Así mismo, debido a la importancia mostrada en temas relacionados con la alimentación (2 preguntas), se incluye una sexta dimensión o atributo denominado "Alimentación". En la cuarta parte, por último, se formula una pregunta, con la que se busca evaluar cuál es el grado de satisfacción global. Se eligió una escala del 1 al 7, siguiendo los parámetros establecidos en el modelo SERVPERF, considerándose de 1 a 4 insatisfacción y de 5 a 7 satisfacción, tal como se muestra en la tabla 1. Se realizó una prueba piloto a cinco adultos mayores de la residencia para mayores, objeto de este estudio, para de este modo poder efectuar las correcciones oportunas al instrumento y obtener la encuesta final ad hoc. Todos los encuestados fueron entrevistados de forma personal y privada, por el mismo entrevistador, y el tiempo medio que se estuvo con cada uno de ellos fue de 15 y 20 minutos.

Tabla 1. Escala de evaluación y su correspondencia con el grado de satisfacción

\begin{tabular}{|c|c|c|c|c|c|c|}
\hline Nada de acuerdo & Casi nada & En desacuerdo & $\begin{array}{l}\text { Ni de acuerdo } \\
\text { ni en desacuerdo }\end{array}$ & Acuerdo & Muy de acuerdo & $\begin{array}{l}\text { Totalmente de } \\
\text { acuerdo }\end{array}$ \\
\hline 1 & 2 & 3 & 4 & 5 & 6 & 7 \\
\hline $\begin{array}{l}\text { Totalmente } \\
\text { insatisfecho }\end{array}$ & Bastante insatisfecho & Insatisfecho & Conforme & Satisfecho & Bastante satisfecho & Totalmente satisfecho \\
\hline \multicolumn{4}{|c|}{ Insatisfecho } & \multicolumn{3}{|c|}{ Satisfecho } \\
\hline
\end{tabular}


Este estudio fue realizado durante los meses de mayo y junio de 2017. Para la evaluación de la información obtenida de las encuestas de satisfacción se utilizó el programa estadístico SPSS Statistics versión 23. Se llevó a cabo análisis descriptivo, distribución de frecuencias, medias y desviación estándar, para cada una de las preguntas de forma individual, por dimensión y la evaluación global de la satisfacción.

\section{RESULTADOS}

La edad de los residentes oscilaba entre los 50 y los 100 años, el 55,17\% de los encuestados era de sexo femenino y el 44,83 , masculino. Con respecto al motivo por el cual se encontraban viviendo en la residencia, prevaleció por motivos de salud $(55,17 \%)$, seguido de por motivos familiares $(25,86 \%) y$, por último, por voluntad propia $(25,86 \%)$.
Se utilizó una escala de evaluación del 1 al 7, para evaluar la percepción que había tenido el entrevistado y su correspondencia según el grado de satisfacción, donde se evidenció que de las medias obtenidas por cada una de las preguntas, se presentó un mayor grado de satisfacción en los horarios de visitas $(6,54)$, la amabilidad y respeto de los empleados $(6,19)$, la comodidad y confortabilidad de las habitaciones $(5,95)$, la confianza de los empleados $(5,95)$, su disponibilidad a ayudar $(5,91)$ y el conocimiento para desarrollar sus funciones $(5,90)$, mientras que las preguntas que causaron insatisfacción fueron las relacionadas con la alimentación, la disponibilidad de menús diarios adaptados a las necesidades de los residentes $(4,19)$ y la variedad de bebidas y comidas $(4,34)$, y el miedo que sienten los residentes a perder algún enser, joya, ropa, entre otros $(4,95)$. Con respecto al resto de las preguntas, fueron catalogadas dentro de los rangos calificados como de satisfacción (tabla 2).

Tabla 2. Relación de las medias por cada una de las preguntas

\begin{tabular}{|c|c|c|c|}
\hline Dimensión & Preguntas & Media & $\begin{array}{l}\text { Desviación } \\
\text { estándar }\end{array}$ \\
\hline \multirow{3}{*}{$\begin{array}{l}\text { Elementos } \\
\text { tangibles }\end{array}$} & 1. ¿Las habitaciones donde usted reside son cómodas y confortables? & 5,95 & 1,594 \\
\hline & 2. ¿Las instalaciones comunes de la residencia son cómodas y confortables? & 5,41 & 1,758 \\
\hline & 3. ¿Los elementos materiales (mobiliario, ropa de cama, utensilios) relacionados con el servicio son visualmente atractivos? & 5,43 & 1,948 \\
\hline \multirow{4}{*}{ Fiabilidad } & 4. Cuando en la residencia los empleados le prometen hacer algo, ¿lo hacen? & 5,55 & 1,846 \\
\hline & 5. Cuando se le presenta un problema, ¿se interesan en resolverlo? & 5,50 & 1,894 \\
\hline & 6. No tiene que preguntar cosas más de una vez, antes de que sean realizadas & 5,16 & 1,705 \\
\hline & 7. Los empleados de la residencia no cometen errores cuando le brindan los diferentes servicios & 5,64 & 1,703 \\
\hline \multirow{4}{*}{$\begin{array}{l}\text { Capacidad } \\
\text { de } \\
\text { respuesta }\end{array}$} & $\begin{array}{l}\text { 8. ¿Recibe suficiente información sobre asuntos de la residencia que le afecten (plan de atención, actividades } \\
\text { de entretenimiento, diversión)? }\end{array}$ & 5,31 & 1,894 \\
\hline & 9. Cuando el personal promete venir dentro de cierto tiempo, ¿lo realiza? & 5,60 & 1,643 \\
\hline & 10. ¿El personal siempre está dispuesto ayudar cuando usted lo necesita? & 5,91 & 1,689 \\
\hline & 11. Cuando usted tiene dudas o quiere saber cualquier cuestión sobre la residencia, ¿se la solucionan inmediatamente? & 5,62 & 1,631 \\
\hline \multirow{5}{*}{ Seguridad } & 12. ¿El comportamiento de los empleados le infunde confianza? & 5,95 & 1,711 \\
\hline & 13. ¿Piensa usted que el personal respeta adecuadamente su intimidad (por ejemplo, en el aseo personal)? & 5,84 & 1,843 \\
\hline & 14. ¿El personal de la residencia es amable y respetuoso? & 6,19 & 1,370 \\
\hline & $\begin{array}{l}\text { 15. Profesionalismo (conocimiento) de los empleados (cuidadores, médicos, enfermeras, auxiliares) en el desarrollo de sus } \\
\text { funciones }\end{array}$ & 5,90 & 1,594 \\
\hline & 16. ¿Tiene miedo a que le roben sus enseres, joyas, ropa, etc.? & 4,95 & 2,259 \\
\hline \multirow{4}{*}{ Empatía } & $\begin{array}{l}\text { 17. ¿Tienen en cuenta sus hábitos personales (horarios al levantarse, ir a la cama, comidas, tiempo libre, ver televisión, } \\
\text { entre otros)? }\end{array}$ & 5,78 & 1,826 \\
\hline & 18. ¿Los horarios de visitas de sus familiares son adecuados y convenientes? & 6,54 & 1,240 \\
\hline & 19. ¿Cree que el personal de la residencia le dedica el tiempo adecuado para su cuidado y su atención personal? & 5,66 & 1,792 \\
\hline & 20. ¿Lo reconfortan (animan) cuando se siente triste 0 solo? & 5,72 & 1,755 \\
\hline \multirow{2}{*}{$\begin{array}{l}\text { Alimenta- } \\
\text { ción }\end{array}$} & 21. ¿La residencia cuenta con una variedad de comida y bebidas? & 4,34 & 1,943 \\
\hline & $\begin{array}{l}\text { 22. ¿La residencia dispone de menús diarios adaptados a las necesidades dietéticas y nutricionales de los residentes } \\
\text { (desayuno, comida, cena, meriendas)? }\end{array}$ & 4,19 & 2,039 \\
\hline $\begin{array}{l}\text { Pregunta } \\
\text { general }\end{array}$ & 23. De forma general, ¿cómo califica usted el servicio que ofrece la residencia? & 5,86 & 1,791 \\
\hline
\end{tabular}


Tabla 3. Comparación entre la media y el nivel de importancia

\begin{tabular}{|l|c|c|c|}
\hline \multicolumn{1}{|c|}{ Dimensión } & Media & $\begin{array}{c}\text { Desviación } \\
\text { estándar }\end{array}$ & $\begin{array}{c}\text { Nivel de } \\
\text { importancia }\end{array}$ \\
\hline Elementos tangibles & 5,60 & 1,714 & 1 \\
\hline Fiabilidad & 5,46 & 1,787 & 3 \\
\hline Capacidad de respuesta & 5,61 & 1,720 & 4 \\
\hline Seguridad & 5,77 & 1,813 & 2 \\
\hline Empatía & 5,92 & 1,700 & 5 \\
\hline Alimentación & 4,27 & 1.984 & 6 \\
\hline
\end{tabular}

Fuente: elaboración propia

La alimentación fue la dimensión considerada como más importante y la que obtuvo un grado de insatisfacción relevante, ya que le otorgaron la calificación más baja de la encuesta. Por el contrario, los elementos tangibles fueron seńalados como los menos importantes, posiblemente asociados al grado de satisfacción seńalado. Aunque esta dimensión no obtuvo la mayor puntuación de la encuesta, la mayoría de los encuestados se encuentran satisfechos. Con respecto a la clasificación del resto de las dimensiones, todas ellas son asociadas de un modo mayor o menor con los empleados, por lo que en el momento de clasificarlas se observa que todas eran importantes, ya que los empleados son vistos como una de las grandes fortalezas de la residencia y en la calificación obtenida por cada una de estas dimensiones se evidencia satisfacción, tal como se recoge en la tabla 3 .

\section{DISCUSIÓN}

Como primera medida, al indagar acerca del motivo por el que se encuentran viviendo en la residencia para mayores, aunque los resultados arrojaron como primera causa motivos de salud, continuando con familiares y finalizando con voluntario, los encuestados, como causa principal del motivo de permanencia en la residencia, lo asocian a su grado de dependencia, ya que no pueden valerse por sí solos. De ahí se derivan secundariamente las causas anteriormente expuestas, por problemas de salud, lo asocian a las diferentes patologías que padecen, familiares, a que sus seres queridos por diferentes motivos no pueden hacerse cargo de ellos, y voluntariamente, ya que deciden de manera autónoma ingresar en este tipo de instituciones, y de esta forma no ser una carga para ninguno de sus familiares o amigos.

En lo referente a las dimensiones evaluadas, la empatía obtuvo de manera conjunta la mejor percepción por parte de los encuestados, donde el horario de visitas de los familiares fue percibido como la pregunta que causa mayor satisfacción a los residentes, mientras que la dimensión alimentación fue la menor. Al clasificarlas según el nivel de importancia referido, se observa que la alimentación es la dimensión que se percibe como la más importante, lo que se puede relacionar con el grado de insatisfacción que se percibe por parte de sus residentes, y al deseo de que esta sea mejorada. Continuando en orden de importancia, la empatía, capacidad de respuesta, fiabilidad, seguridad y, por último, los elementos tangibles fueron catalogados como los menos importantes, lo que puede estar relacionado con la satisfacción que se siente por ellos. Edys $^{16}$ confirma esta circunstancia, señalando en su estudio que, entre los atributos menos importantes, evaluados en residencias para mayores, se encuentran las instalaciones físicas, tanto sus habitaciones como zonas comunes, mientras los atributos más importantes incluyen profesionalismo y atento cuidado de los residentes, amabilidad y respeto en rela- ción con el residente y su familia. Así mismo, Lapré10 destaca que entre los aspectos que contribuyen a la percepción de calidad en las residencias para mayores se encuentra cómo responde el personal a las peticiones de los residentes, referente a la hospitalidad, aspectos como una amplia elección de comida y bebidas, actividades a lo largo del día, la cortesía en la interacción entre el personal y los residentes, y la aproximación de los empleados hacia los residentes.

Respecto a los elementos tangibles, Gutiérrez et al. ${ }^{17}$ consideran a estos como factores indispensables para lograr el bienestar de los residentes, resaltando las habitaciones individuales y su limpieza. Molina et al. ${ }^{18}$ señala que los elementos físicos, como son las habitaciones, constituyen el área propia y personal de los residentes, equivalente a su hogar. El presente estudio corrobora lo anteriormente expuesto, donde las habitaciones son vistas como una de las grandes fortalezas de la residencia, por ser espaciosas, confortables, cómodas y contar con baño en su interior. No obstante, la necesidad de compartirlas o su ubicación dentro de la residencia son causas que se perciben como insatisfacción; con respecto a los elementos materiales, referidos como el mobiliario, utensilios, ropa de cama, entre otros, aunque son percibidos de manera satisfactoria por sus residentes, la inconformidad mostrada se asocia con el deterioro y la cantidad de los utensilios del comedor, ya que son considerados insuficientes para atender los requerimientos de todos los residentes, así como manchas encontradas en ropa de cama y toallas. Sobre las áreas comunes, algunos autores como Rocha et al. ${ }^{19}$ señalan la falta de higiene de estas áreas como causa de insatisfacción. Este estudio corrobora lo anteriormente expuesto, aunque específicamente en los bańos comunes, que son asociadas a la falta de higiene, catalogados como insuficientes para la cantidad de residentes, además de encontrarse en mal estado.

Los empleados son vistos como una de las grandes fortalezas con que cuenta la residencia, por su dedicación, respeto y amabilidad, disponibilidad, confianza y conocimiento en el desarrollo de sus funciones, haciéndolos sentir como si estuvieran en casa. Podemos comprobarlo en diferentes estudios que señalan a los empleados como un factor que puede afectar de forma positiva a la percepción de la calidad en el cuidado de los residentes ${ }^{17,20}$, siendo uno de los atributos más importantes en la evaluación de la calidad en residencias para mayores ${ }^{16}$. Sin embargo, en cuanto a la cantidad, los consideran insuficientes en relación con el número de residentes, ya que, por su grado de dependencia, demandan cuidados constantes, que no pueden ser realizados inmediatamente, ya que los empleados se encuentran ocupados tratando de suplir las necesidades de otros residentes, lo que genera esperas prolongadas cuando solicitan o les prometen algo.

En lo que concierne al respeto de la intimidad por parte de los empleados, algunos estudios lo refieren como una de las grandes causas de insatisfacción ${ }^{17,19}$. Este estudio, por el contrario, muestra satisfacción por parte de los entrevistados, caracterizándose por el respeto que muestran los empleados a la intimidad de los residentes. No obstante, la insatisfacción referida por algunos encuestados podría estar asociada a su grado de dependencia y patologías, ya que en algunos casos los empleados deben entrar a las habitaciones o baños de los residentes para corroborar cómo se encuentran.

Sobre las causas de insatisfacción, el miedo que pueden sentir los residentes a que les roben sus enseres, joyas, entre otros, es asociado a casos puntuales, donde algunos de los residentes afirman haber perdido algún tipo de enser, ropa, etc., o mencionan haber oído hablar sobre la pérdida de algún objeto por parte de otros residentes.

Respecto a la alimentación, Rocha et al. ${ }^{19}$ concluyen que, aunque se cuente con una variedad de menús, la falta de elaboración de las comidas puede afectar a la satisfacción de las personas de la tercera edad. Este estudio reafirma lo anteriormente expuesto, asociado a la baja calidad, 
variedad, confección y sabor de los alimentos, así como a la falta de variedad de bebidas, ya que algunos de los entrevistados enfatizan el hecho de que se encuentran pagando por estos servicios, seńalando que el servicio de alimentación debería mejorar. De forma adicional, apuntan las pequeñas porciones en relación con frutas y pan.

Finalmente, en general, el servicio que ofrece la residencia es percibido como satisfactorio, donde la mayoría de los encuestados la ven como su hogar y en donde terminarán sus días. Por este motivo, prestar servicios de alta calidad que satisfagan sus necesidades y expectativas se debe convertir en un reto diario, que debe contar con su participación para conseguir su satisfacción, ya que, como seres humanos, la satisfacción debe convertirse en uno de sus objetivos ${ }^{21}$.

\section{- CONCLUSIONES}

Las personas de la tercera edad que residen en residencias para mayores son el canal más importante para obtener información sobre cuestio- nes relacionadas con la residencia y se convierten en una herramienta clave para la mejora de la calidad en la prestación del servicio que se ofrece.

Los residentes de una residencia para mayores, privada, ubicada en una población de la Comunidad Valenciana, se encuentran satisfechos con el servicio ofrecido (83,71\%).

Entre las principales causas de satisfacción señaladas por los residentes se encuentran: las habitaciones, consideradas cómodas y confortables; los empleados, caracterizados por su respeto y amabilidad, disponibilidad a ayudar, confianza y conocimiento para el desarrollo de sus funciones y, por último, los horarios de visita de sus familiares.

Las causas más importantes de insatisfacción de los residentes son: la alimentación, asociada al hecho de que hacen un desembolso importante por estos servicios, y el miedo que pueden sentir a que les puedan robar alguno de sus enseres

\section{Conflicto de intereses}

Los autores declaran no tener ningún conflicto de intereses.

\section{- BIBLIOGRAFÍA}

1. Parasuraman A, ZeithamI VA, Berry LL. SERVQUAL: A Multiple-Item Scale for Measuring Consumer Perceptions of Service Quality. Journal of Retailing. 1988; 64(1):12-40.

2. Baggs SC, Kleiner BH. How to measure customer service effectively. Managing Service Quality. An International Journal. 1996;6(1): 36-9.

3. Parasuraman A, ZeithamI VA, Berry LL. A Conceptual Model of Service Quality and its Implication for Future Research (SERVQUAL) Journal of Marketing. 1985:49:41-50.

4. Brady MK, Cronin J, Brand RR. Performance-only measurement of service quality: a replication and extension. Journal of Business Research. 2002;55(1):17-31.

5. Landrum H, Prybutok VR, ZhangA X. A comparison of Magal's service quality instrument with SERVPERF. Information \& Management 2007:44(1):104-13.

6. Cronin Jr JJ, Taylor SA. Measuring service quality: A reexamination and extension. The Journal of Marketing. 1992:56(3):55-68

7. Abellán GA, Ayala GA, Pujol RR. Un perfil de las personas mayores en España, 2017. Indicadores estadísticos básicos. Madrid Informes Envejecimiento en red $n^{0}$ 15. (internet) 2017 Ene. [citado 21 de abril de 2017]. Disponible en: http://envejecimiento.csic.es/documentos/documentos/enredindicadoresbasicos13pdf
8. Rojas OMJ, Toronjo GA, Rodríguez PC, Rodríguez RJB. Autonomía y estado de salud percibidos en ancianos institucionalizados. Gerokomos. 2006:17(1):6-23.

9. Muñoz PA, Martínez CG, Martínez EC, Escámez IM, Martínez IA Muñoz FA. Sistemas de gestión de calidad en el área de enfermería un antes y un después en el mundo de las residencias para persona mayores. Eur J Investig Health Psychol Educ. 2015;5(1):99-108.

10. Lapré FAL. Service quality in nursing homes. A construct, measu rement and performance model to increase client focus (Doctora dissertation): University of Bradford: 2013

11. San Miguel Osaba E. Gestión de la calidad y marketing en los centros residenciales para personas mayores. (Tesis doctoral): Universidad del País Vasco; 2011

12. Lowe JT, Lucas JA, Castle NG, Robinson JP, Crystal S. Consumer Satisfaction in Long-Term Care: State Initiatives in Nursing Homes and Assisted Living Facilities. The Gerontologist. 2003:43(6):883-96.

13. Basantes, AR, Vinueza JV, Coronel SJ. Niveles de satisfacción de los usuarios del Hospital Andino alternativo de Chimborazo. Revista Ciencia UNEMI. 2016;9(10):90-100.

14. Ibarra MLE, Casas MEV. Aplicación del modelo Servperf en los centros de atención Telcel, Hermisollo: Una medición de la calidad en el servicio. Contaduría y Administración. 2015;60(1):229-260.
15. Lobo A, Saz P, Marcos G, Día JL, de la Cámara C, Ventura T, et al. Revalidación y normalización del Mini-Examen Cognoscitivo (primera versión en castellano del Mini-Mental Status Examination) en la población general geriátrica. Med Clin (Barc). 1999;112(20):767-74.

16. Ejdys J. Prospective quality attributes of nursing home care senvices. In: Published by VGTU Press, 2016: 9th International Scientific Conference. Bussiness and Management 2016. May 12-13; Vilnius, Lithuania; p. 1-10

17. Gutiérrez J, Linares AM, Grande GL. El concepto de bienestar en las residencias de personas mayores. Evidentia: Revista de enfermería basada en la evidencia. 2011:8(36):1

18. Molina SC, Meléndez MJC, Navarro PE. Bienestar y calidad de vida en ancianos institucionalizados y no institucionalizados. Anales de Psicología. 2008:24(2):312-9.

19. Rocha RM, Álvarez LME, Bayarre VHD, Rosell P, Yurina A, Delgado, RY, et al. Satisfacción de usuarios y prestadores de servicio en relación con la atención en el hogar de ancianos "General Peraza". Revista Habanera de Ciencias Médicas. 2015;14(4):496-505.

20. Vigdis AG, Liv BF. "Nursing home care quality: a cluster analysis", Int J Health Care Qual Assur. 2017;30(1):25-36.

21. Cano GL. Análisis del grado de satisfacción de los usuarios que participan en el Programa de Animación Sociocultural de la Residencia San José de Santovenia de Pisuerga (Trabajo fin de grado): Universidad de Valladolid; 2012 